\title{
ASS senkt Sterberisiko beim Prostatakarzinom
}

\section{Seit Jahren schon mehren sich die Hinweise, dass Antikoagulantien das Wachstum und die Metastasierung von Tumorerkrankungen hemmen können. Klinische Daten aber fehlten bislang. Nun hat eine Studie an 6.000 Männern mit Prostatakarzinom tatsächlich einen Schutz vor allem durch Acetylsalicylsäure (ASS) belegt.}

Z unehmend wird klar, dass es eine Verbindung zwischen Krebserkrankungen und dem antikoagulären System gibt: So leiden Tumorpatienten besonders häufiger unter Thrombosen; umgekehrt haben Patienten, bei denen eine Diagnose für idiopathische venöse Thrombosen gestellt wurde, ein erhöhtes Risiko für Krebserkrankungen. Aus diesem Zusammenhang ergibt sich auch, dass Antikoagulantien womöglich Wachstum und Metastasierung von $\mathrm{Tu}-$ moren hemmen können. Dafür gibt es inzwischen auch experimentelle Belege; klinische aber sind rar gesät.

In einer umfassenden Studie an knapp 6000 Männern mit Prostatakarzinom sollte daher konkret untersucht werden, ob der Einsatz von Antikoagulantien mit dem Risiko assoziiert ist, an Prostatakrebs zu sterben. Die Daten der Männer entstammten der „Cancer of the Prostate Strategic Urologic Research Endeavor"-Datenbank. Bei allen Probanden lag ein lokalisiertes Adenokarzinom der Prostata vor, das bereits mittels radikaler Prostatektomie oder Radiotherapie behandelt worden war. $37 \%$ der Probanden $(n=2175)$ bekamen Antikoagulantien (Wafarin, Clpidogrel, Enoxaparin und/oder Acetylsalicylsäure. Die Prostatakrebs-spezifische Mortalität wurde ermittelt.

Nach einem mittleren Follow-up von 70 Monaten war das Risiko, an Prostatakrebs zu sterben, in der Gruppe, die Antikoagulantien erhielt, im Vergleich mit der Gruppe ohne Antikoagulantien signifikant geringer (3 vs. $8 \%$ nach zehn Jahren). Das Risiko für ein Rezidiv und für Knochenmetasen war ebenfalls signifikant niedriger. In einer Subgruppenanalyse war die Senkung der prostatakrebsspezifischen Mortalität am prominentesten bei Patienten mit Hochrisikoerkrankung (4 vs. 19\% nach zehn Jahren). Der Nutzen zugunsten der Antiko- agulantien blieb unabhänggg von der Behandlungsmodalität wie Radiotherapie oder Prostatektomie bestehen.

Eine Analyse der verabreichten Antikoagulantien legte nahe, dass die $\mathrm{Re}$ duktion der prostatakrebsspezifichen Mortalität primär mit ASS assoziiert war. Eine Multivarianzanalyse ergab, dass das Nutzen von ASS mit einer niedrigeren prostatakrebsspezifischen Mortalität assoziiert war (adjustierter Risikoquotient $[\mathrm{HR}]=0,43$ ).

Fazit: Eine Therapie mit Antikoagulantien, vor allem mit Acetylsalicylsäure, ist bei Männern, die wegen eines Prostatakarzinoms eine Bestrahlung oder eine Prostatektomie erhielten, mit einem reduzierten Risiko assoziert, an dem Prostatakarzinom zu versterben Die Assoziation war am deutlichsten bei Patienten mit besonders aggressiver Erkrankung.

Christina Berndt

Choe KS et al. Aspirin use and the risk of prostate cancer mortalitay in men treated with prostatectomy or radiotherapy. J Clin Oncol. 2012 Aug 27. [Epub ahead of print]
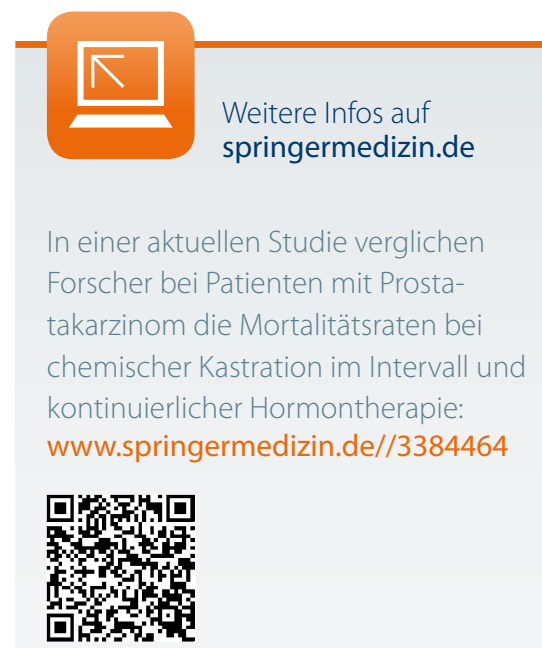

ALIMTA $^{\circledR} 100 \mathrm{mg}$ bzw. $500 \mathrm{mg}$ Pulver zur Herstellung eines Konzentrates zur Herstellung einer Infusionslösung. Wirkstoff: Pemetrexed. Zusammensetzung: Jede Durchstechflasche enthält $100 \mathrm{mg}$ bzw. $500 \mathrm{mg}$ Pemetrexed (als Pematrexeddinatriun. Nach Aülösung enth jede Durchstechflasche $25 \mathrm{mg} / \mathrm{ml}$ Pemetrexed. Sonstige Bestandteile: Mannitol, Salzsäure, Natriumhydroxid (enthält ca. $11 \mathrm{mg}$ bzw. $54 \mathrm{mg}$ Natrium). Anwendungsgebiete: Malignes Pleuramesotheliom: In Kombination mit Cisplatin zur Behandlung von chemonaiven Patienten mit Pur first-line Therapie von Patienten mit lokal fortgeschrittenem oder metastasiertem nicht-kleinzelligen Lungenkarzinom außer bei überwiegender plattenepithelialer Histologie. In Monotherapie für die Erhaltungstherapie bei lokal fortgeschrittenem ode metastasiertem nicht-kleinzelligen Lungenkarzinom Ber bei uberiegender platte Histologie bei Patienten, deren Erkrankung nach einer platinbasierten Chemotherapie nicht unmittelbar fortgeschritten ist. In Monotherapie zur Behandlung in Zweitlinientherapie von Patienten mit lokal fortgeschrittenem oder metastasiertem nichtkleinzelligen Lungenkarzinom außerbeiübenwiegender plattenther off ode einen der sonstigen Bestandteile, Stillen, gleich zeitige Gelbfieberimpfung. Nebenwirkungen: Sehr häufig: Neutrophile/Granulozyten, Leukozyten, Hämoglobin, Thrombozyten erniedrigt, Diarrhoe, Erbrechen, Stomatitis/Pharyngitis, Übelkeit, Appetitverlust, Obstipation, Müdigkeit, Neuropathie Clearance erniedrigt, Hautrötung/Abschuppung, Haarausfall. Häufig: Erkrankungen der Augenoberfläche (einschl. Konjunktivitis), verstärkter Tränenfluss, Dyspepsie/Sodbrennen, Mukositis, Dehydratation, Geschmacksstörung, Infektion, Sepsis (manchmal tödlich verlaufend), Fieber febrile Neutropenis AST (SGOT), Gamma-GT erhöht, ALT (SGPT) erhöh Juckreiz, allergische Reaktionen/Überempfindlichkeitsreakt ionen, Brustschmerzen, motorische Neuropathie, sensorische Neuropathie, multiformes Erythem, Bauchschmerzen, Ödeme, verminderte glomeruläre Filtrationsrate, Nierenversagen, Schmerzen. Gelegentlich: Arrhythmie (u. a supraventrikuläre), schwenticg: Arhythmie (u.a supraventikulare), kuläre Ereignisse, einschließlich Myokardinfarkt, Angina pectoris, zerebrovaskulärer Insult und transitorische ischämische Attacken (die meisten dieser Patienten, hatten vorbestehende kardiovaskuläre Risikofaktoren), Panzytopenie, Colitis (einschließlich intestinale und rektale Blutungen, manchmal ticlich veraufende, intestine Perforation, tödlich verlaufende interstitielle Pneumonitis mit respiratorischer Insuffizienz, Ösophagitis/StrahlenÖsophagitis, Strahlenpneumonitis (bei Patienten, die vor, während oder nach ihrer Pemetrexed Therapie bestrahlt wurden), periphere Ischämien (die manchmal zu Nekrosen an den Extremitäten (ühren), Lungenembole. Selten: Hepatils, m fuhren), Lungenembole, Seltrien HögRecall (bei Patienten, die vorher eine Strahlentherapie erhalten hatten), bullöse Erkrankungen einschließlich Stevens-Johnson Syndrom und toxischer epidermale Nekrolyse (in einigen Fällen tödlich verlaufend), hämolytische Anämie, anaphylaktischer Schock. Warnhinweise: Vorbehandlung Begleitherapie mit Folsäure und Vitamin $B_{12}$ sc Kortikosteroid notw Kortikosteroid notwendig, weitere Warnhinweise s. Fachinformation. Verschreibungspflichtig. Pharm. Unternehmer: Eli Lilly Nederland B.V., Grootslag 1-5, 3991 RA, Houten, Niederlande. Vertrieb: Lilly Deutschland $\mathrm{GmbH}$, Werner-Reimers-Straße 2-4, 61352 Bad Homburg, Deutschland. Stand der Information: Juli 2012 DEALM00932C 